\author{
JOE O'HARA \\ Dublin City University, Ireland \\ GUNILLA HOLM \\ University of Helsinki, Finland
}

\begin{abstract}
Drawing on their personal experiences, the authors reflect on the relationship between the European Educational Research Association (EERA) Council and the National Educational Research Associations (NERAs). The article will argue that while much of the work undertaken by the EERA Council is hugely valuable, at times it can be difficult to see a causal link between discussions and decisions at this level and changes in governance and practice at the level of national associations. Having said that, it is hoped that the article will give an idea of issues that emerged and challenges that might yet need to be faced in the EERA Council. These included the impact of changes in governance structures on the way EERA interacts with NERAs and other constituent groups. In addition, there has also been an ongoing debate around the definition of European in the context of educational research and the connected challenges of the identification and operationalisation of a core set of values to underpin this endeavour. Finally, the article explores the manner in which EERA Council as a representative body of national associations helped shape the processes whereby the association interacted with a range of external partners.
\end{abstract}

\title{
Introduction
}

The authors of this article have collectively spent over a decade as members of the European Educational Research Association (EERA) Council. Joe O'Hara was the Irish representative from 2008 to 2013 and Gunilla Holm has been the Finnish representative since 2009. The reasons for the extended period of their respective presence on Council are both prosaic and instructive. Both represent comparatively small though active associations that operate in national contexts and have undergone significant changes over the past decade. Ireland and Finland have seen educational funding models change, have witnessed - albeit in divergent ways - the impact of external evaluative systems such as the Programme for International Student Assessment (PISA) on perceptions of quality in education, and have been forced to explore alternatives to current ways of structuring basic educational provision. As educational research associations, the Educational Studies Association of Ireland (ESAI) and the Finnish Educational Research Association (FERA) have sought to reflect on and, where possible, influence these changes, with mixed results.

Against this background, membership of EERA became an interesting - and in theory at least - useful conduit for bringing a broader European perspective to the issues being addressed nationally. In practice, however, ongoing reductions in funding for educational research, along with the difficulties of maintaining membership in a period of significant financial upheaval coupled with greater demands on the time of educational professionals, have seen both associations rely on the same individuals to represent them for extended periods. In the context of this article, the 
comparative length of service on Council has given both authors a particular perspective on the role of National Educational Research Associations (NERAs) in EERA, the relationship between NERAs and Council and the manner in which this has evolved in recent years. It has also allowed them to critique the operation of EERA Council from a position of genuine understanding, as their presence at 15 Council meetings totalling approximately 200 hours of discussion has at the very least allowed them come to a sense of how Council sees itself, if not necessarily of how the rest of EERA sees Council. From the outset readers should note that this article seeks to give an impressionistic overview of some key issues that arose in the course of the last 5-6 years in the life of the EERA Council. It does not pretend, nor seek, to be comprehensive; indeed, other Council members might have a completely different set of memories and priorities. The article will explore the relationship between Council, networks and NERAs. It is hoped that it will also give an idea of issues that emerged and challenges that might yet need to be faced in the EERA Council. Finally, the authors clearly view EERA Council as being the collective body of National Educational Research Associations, and discussions of Council structures, activities and challenges in the following pages should be viewed in this light.

\section{The Structure of EERA}

EERA has 31 national or regional member associations. Each association has its own representative on the EERA Council. Additional members on the Council are the president, secretary-general, treasurer, network representatives and the Emerging Researchers' Group representative. The research is organised into 31 networks, with an additional non-thematic group, the Emerging Researchers' Group (ERG). The annual EERA conference, the European Conference on Educational Research (ECER), is organised thematically around the networks. The ERG organises a preconference for emerging researchers. EERA also has its own journal, the European Educational Research Journal.

One of the more interesting challenges for any representative of an association attending EERA Council for the first time is to work out what it is and how it operates. In theory it is simple; the governance structure is explained as follows:

EERA is governed by the Council and the Executive Board. Council consists of the

representatives of the member associations and the co-opted council members - the Networks'

Representative on Council, the editor of EERJ and the Convenor of the Emerging Researchers'

Group. Since June 2010 Council has the power of the Annual Assembly.

The Executive Board consists of the President, the Treasurer and the Secretary General and shall implement the Council resolutions and submit to the Council proposals concerning the achievement of the association's purpose. (EERA, 2014)

The clarity provided here masks a degree of confusion around some key definitions. Take, for example, the statement that 'Council consists of the representatives of the member associations' while in theory a comparatively straightforward observation, in practice it overlooks the reality that there are a variety of different types of research associations represented - from what the EERA website chooses to identify as 'General Educational Research Associations' (EERA, 2014), to regional associations which represent different cultural and political viewpoints on provision within national contexts, to tightly focused research associations focusing on one particular aspect of educational provision. The practical result of this is that some countries are represented by multiple associations, most being represented by one association and others being represented by supranational organisations. While this multiplicity in representation types is not necessarily problematic in theory, in practice it results in a situation where defining the 'association's purpose' (EERA, 2014) can be a complex and at times contentious process. An additional complication is the increasing size of Council. What began as group of 19 has now developed into a body where upwards of 40 people can be present at each meeting (EERA, 2010, pp. 3-4).

To be fair, both Council and the wider association have recognised the difficulties posed by this issue, and at the 2012 Annual General Assembly (AGA) a new set of criteria for membership was agreed upon and entered into the Constitution (EERA, 2011). These criteria require, among other things, that: 
- Prospective members represent general educational research associations operating at a national level;

- Where there is already representation at a national level, any new member seeks to create a formal relationship with the national association which obliges them 'to work toward joint membership under the umbrella of a national organisation for general educational research';

- The representative status of members of Council prior to 2012 remains unchanged.

The new requirements go some way to addressing the future needs of Council but there are still significant legacy issues remaining. The differing representational levels and types of organisational inputs lead to a range of often conflicting responses to emerging policy or structural decisions - and some of the more pressing of these will be discussed later.

As the number of member associations is increasing another issue is emerging - namely, what is meant by a European association? Should, for example, the member list of the Council of Europe be used as tool for deciding what association is European or should the criterion be that the country is geographically located in Europe? The authors would argue that it will become necessary for EERA as a broad organisation and Council as a representative body to address this issue as a matter of urgency. To date, and understandably, the argument as to European identity has been one that has been dominated by geographic and technical criteria. While this mode of definition has been useful, there are now broader questions that need to be addressed. For example, can national associations from countries with autocratic or quasi-democratic political cultures be accepted as members? And if not, how can EERA support independent educational research in such countries? Does European stand for certain values like democracy, independence and non-discrimination? Do the member associations have to stand for these values? We will return to this issue at a later stage, addressing it in the context of particular challenges that emerged during ECER 2013.

The sheer size of the Council meeting has also resulted in the emergence - by necessity - of a range of ancillary structures, including the Executive, which, it could be argued, reduces the ability of ordinary Council members to shape policy in the manner originally envisaged. This is not to suggest that the Executive seeks to limit discussion, merely that by taking on a filtering function 'submit to the Council proposals concerning the achievement of the association's purpose' - that it guides the prioritisation of policy in a manner that did not exist prior to its creation. In reality the creation of the Executive is probably the most significant change to have occurred during our time on Council and to an extent its impact on the way NERAs engage with EERA is still evolving. In the early part of our respective periods on Council a significant proportion of the discussion that took place was procedural in nature, dealing with the minutiae of governance - much of which was of little interest to many sitting around the table. While not wishing in any way to diminish the importance of having strongly democratic and inclusive governance structure, the necessity of micro-managing each decision and putting it to a vote of all members was hard to justify. It was particularly problematic for new members who were often being asked to make decisions in areas that required either specialist knowledge or a long history of engagement with EERA. The creation of additional space at Council meetings has allowed NERAs to begin the process of considering how EERA might shape discourse and discussion around key themes in educational research. Arguably, neither the NERAs nor the Executive have found the mechanism to facilitate this in a manner that will lead to significantly enhanced engagement at national levels; however, the emergence of a range of focused discussions, presentations and other innovations has seen Council at least begin the journey towards a new mode of operation in this area.

In addition to the way that Council structures its work, a second issue that has emerged in recent years is the confirmation of English as the lingua franca of all EERA-related communications. While there are many and varied practical reasons as to why this is so, it does result in Council discussions favouring those who are confident expressing themselves in English. At times this can lead to a situation where the articulation of complex national perspectives - one of the original ideas of EERA (EERA, 2010, pp. 3-4) - does not happen to the extent that it might. There have been attempts to address this, particularly through the introduction of formal inputs from each national association on the context within which they work, but the broader issue of the dominance of a particular language and associated worldview is one that remains problematic. The authors would argue that this is an area that needs to be prioritised in future years. At a practical 
level, consideration might be given to providing translation facilities for key discussions or, accepting that this might not be feasible, to at least providing key policy documents in a range of national languages. This suggestion might also have the added benefit of allowing NERAs to disseminate key policy documents at a national level in a manner that would ensure greater penetration across a range of educational communities. In addition to this, a more structured approach to the way in which NERAs are encouraged to network might be considered. The creation of regional and/or linguistically coherent sub-groups of NERAs might provide an opportunity for pre- Council meeting discussions around key policy areas. Assuming that discussions in these fora would be conducted in languages other than English, this structure might allow for a more considered and indeed diverse range of views emerging and being presented at Council.

By 2010 it became obvious that the status quo had become untenable, and following a period of consultation the creation of the aforementioned Executive Board was proposed. Made up of the president, treasurer and secretary-general, this body took an oversight role and was given the power to streamline the decision-making process. In practice this resulted in many of the timeconsuming though important procedural issues being removed from the agenda of Council meetings and, theoretically at least, freed up Council time for the discussion of broader strategic issues. While this process was generally welcomed, there was a sense that something fundamental had changed in terms of governance that went beyond the structural and procedural changes outlined. Some of this may simply have been as a result of the need to become comfortable with new structures and ways of working; however, there was also a sense that a highly consultative and consensual body had perhaps chosen to prioritise efficiency over engagement. It should be emphasised that this was not a universally held position, nor perhaps even a widely held one, but it was raised at the time and for a period subsequently. While acknowledging this, the recent successful management of Council business and the robust governance structure put in place have perhaps vindicated the decision in the eyes of most.

\section{The Relationship between Networks and Council}

One of the more challenging areas for EERA and Council is the relationship between EERA and ECER - and, by extension, between Council and networks. While there is a formal structure for the inclusion of network issues, concerns and perspectives in all Council discussions through the Office of Network Representative, at times there appears to be a disconnect between those on Council and those in the networks. One possible reason for this might be the presence on Council of representatives of associations who have little or no connection with networks. While many Council members have been active in a number of networks, it is clear that some have only a limited understanding of the role of the network in EERA. This has been recognised by Council and there is an attempt at least to provide new members with an overview of the network structure and how it impacts on the operation of EERA. Useful though this is, it cannot address the issue of how the networks and Council actually interact.

Without seeming to over-dramatise the issue, this appears to us to be at the heart of the debate as to the identity of EERA. For many, the dominant entity in EERA is their network, and the most important structure linking them to networks is ECER rather than Council, Executive or, indeed, their NERA. Individuals identify with their network in an EERA context and their network organises their formal interaction - both personal and professional - at ECER. The governing structure of EERA is somewhat distant in this way of viewing the organisation and the important element remains the desire to interact with colleagues from a similar disciplinary background. From a NERA perspective it is arguable that many network members have no real idea as to who represents them on Council, and far less as to what this representation means in practice. Council has been aware of this and a special delegate meeting was held in Berlin in March 2013 to address this question. While there were a number of useful outcomes to this meeting there still remains at the heart the question of how to link individuals with a disciplinary interest to a set of national and sectoral organisations that meet a number of times a year to discuss issues of governance and, when possible, of strategic interest. 
The challenge for Council here is a substantial one. While there is an understanding that the relationship between it and the networks needs to change to become more collaborative and integrated, there is also a sensitivity to the enormous amount of work - both historic and ongoing - that has been undertaken by networks over the years. While some national representatives on Council might seek to be more directive - for example, in areas of network governance, transparency and reporting structures - there is also an understanding that networks have grown organically led by voluntary elected link convenors, have their own cultures and, for the most part, serve their members in a fashion that adds to the quality and quantity of educational research being undertaken in a wide range of disciplinary fields. In this context an attempt to impose greater oversight might be seen as being counterproductive. Having said that, if EERA Council is to continue to address issues in a coherent manner, greater oversight is perhaps inescapable.

\section{Speaking to Ourselves}

Perhaps the greatest impact of the decision to move to an Executive was to give more time at Council meetings to explore issues beyond the procedural. While there were a number of innovations introduced - including strategy discussions, thematic group work, etc. - from the perspective of the creation of an understanding of educational research at a European level arguably the most important was the decision to request a short presentation from each representative on the state of educational research in their country. As is perhaps to be expected, this process highlighted both commonalities and particularities; however, there were some interesting trends that may inform future discussions at Council level.

At what might be considered the most prosaic although essential level, the presentations threw up an almost universal set of organisational issues that impacted on all associations, from the smallest to the largest. These included: the challenges of maintaining membership numbers in a period of economic difficulty; the recruitment of new members from postgraduate students undertaking research in education; the hosting of conferences - whether national or thematic - that allowed researchers to engage in a meaningful dialogue with each other and the wider community; the maintenance of the relevance of the organisation in the face of a changing education landscape; and the provision of outlets for educational research that were not only systemically impactful but also allowed for the diversity of this research to reach as wide an audience as possible. Different associations chose to address these challenges in different ways - and here the comparative size and wealth of the organisations clearly had an impact; however, it was both enlightening and reassuring from the perspective of Council members to see echoes of their own struggles in the experience of others.

This point of organisational size and the role it played in addressing the issues raised at a procedural level was an interesting one. It became clear that one particular advantage offered by a large membership base was the ability to easily organise members into sub-groups around relevant thematic areas. These interest groups offered associations an opportunity to enhance the disciplinary focus of particular sets of discussions while at the same time creating a larger shared space where discrete discussions might be shared. As well as being interesting in an organisational way there was also a sense that the experiences of these associations might offer some useful pathways for further discussion for EERA as a whole as to how the association might deal with the challenge - discussed earlier - of linking networks to Council and Council members in a more meaningful manner. Unsurprisingly, no particular template emerged; however, the process of engaging and developing a shared understanding of the challenges involved did enhance the capacity of Council to examine the issue from an informed perspective - something that a body such as Council should strive for at all times.

In addition to the organisational challenges facing member associations there were other, perhaps more strategic, issues that impacted on large numbers, if not all members. One area of concern to quite a number of the associations presenting was the emerging culture of the measurement of the quality of educational research and the increasing tendency to introduce criteria that were viewed as being contrary to traditional understandings of the role and function of education in a given social context. For example, the elevation of economic relevance and practical 'usefulness' of research to the level of key criteria in the judging of educational quality was seen as 
being particularly problematic in a number of national contexts. A discrete, although connected, challenge was the increased use of quality proxies relating to publications and in particular the emergence of private ranking bodies such as ISI Web of Knowledge and SCOPUS as key arbiters of quality. This development had impacted on most if not all members to some extent and had a significant impact on an increasing number of NERAs. The challenges posed by this movement have been articulated in other spheres and the discussions at Council echoed many of the common ones, including, but not limited to, the narrowing of publishing options, the prioritisation of certain types of research over and above others, the disincentive to publish in formats other than journal articles - and the particular impact that this might have in certain national contexts if the trend were to become more pronounced - and the increasing dominance of the English language medium in all of the measurement indices used.

The latter point was one that was of particular interest to a large number of member associations and was one that had been raised in a number of different contexts in recent years. A number of Council members explicitly highlighted the pressure to publish in English language journals as being a particular challenge and emphasised the potential this had to narrow the range and relevance of research being undertaken at a local, regional and national level. In practice it appears that competence in English has become a gateway to both individual career progression and judgements of institutional quality in a number of countries. This is viewed as being a worrying development in a variety of contexts and one that has the potential to impact on both the diversity of research being undertaken and the range of voices represented at a European level. This process - as mentioned earlier - can be seen as being mirrored at Council level, with English becoming solidified as the formal language of discussion and decision at meetings. In practice this does offer native speakers something of an advantage in these settings and this is a theme that has been raised at different times over recent years. It is noteworthy, though, that none of the current or incoming office holders on Council are from a native English-speaking background, and this may offer a way into a broader discussion of how EERA can represent a range of diverse educational research traditions and cultures in a period where the drive towards homogeneity is becoming more pronounced at both policy and publication level.

A final but significant challenge for virtually all associations presenting at Council is the reduction in research funding that has been experienced in recent years. In some cases this is as a result of scarcity of resources at a national level; in other cases it is due to a substantial reduction in global amounts available to research in higher education. There was a perception that the social sciences in general and education in particular suffered substantially more cuts than other academic areas, and some evidence was alluded to. While it is possible to see a situation developing in future years where these types of cuts might be reversed, the identification of a second strand of funding reduction was considered to be of genuine concern in a range of associations' presentations. Essentially this was seen as being a new approach to funding educational research, which sought to link funding to specific measurable outcomes which were often seen as having a utilitarian focus. While not necessarily a novel approach to funding - indeed, it is one that has dominated European funding models since the 1990s - it was seen as being potentially problematic when applied across the wide range of research areas in education. A further concern was the explicit link made in a number of national contexts between the measurement culture emerging around educational research, the status of that research and the capacity to access funds in order to continue research across the whole range of educational subjects. The discussions that took place at Council around this area were in depth and complex and to an extent can be seen as having a direct impact on what was perhaps the most significant innovation of recent years - the decision by Council to lobby the European Commission directly as part of a concerted campaign to influence the shape and function of the Horizon 2020 programme. It is to this campaign that we will now turn, using it as an exemplar of how the changes at Council provided EERA with an opportunity to change the way in which it sought to engage with key stakeholders in European research.

\section{Speaking to Others}

There have been a number of attempts over recent years to re-position EERA in an increasingly globalised policy and practice environment. The ongoing discussions with the European 
Association for Research on Learning and Instruction (EARLI) around issues of collaboration at Special Interest Group (SIG)/network level as well as at governance level are a good example of this. These conversations have ebbed and flowed with changes in strategic imperatives and, indeed, personnel on both sides, impacting on them to a greater or lesser extent. Much the same could be said around EERA's interaction with the World Educational Research Association (WERA). Ignoring for a moment the wider discussion around the function and need for an organisation such as WERA, the commitment of EERA to bring a diverse perspective to global discussions on educational research and, as importantly, to facilitate other viewpoints through its strategic support of different national and regional associations in WERA is important. Membership of WERA also provided NERAs with an opportunity to examine their own commitment to engagement beyond their national borders. At a practical level this saw some NERAs view EERA as a collective body that could represent them at a 'world' level, which resulted in them choosing not to join WERA as individual associations. For this, group discussions around WERA took on an additional importance as they provided NERAs with an opportunity to bring a collective European voice to the world stage.

Important though the above issues were and continue to be, arguably the campaign to address what were perceived as being significant deficiencies in the conceptualisation, structure and implementation of the Horizon 2020 programme from the perspective of social science research in general and educational research in particular marked a significant change in EERA's engagement with the wider research community. While it would be untrue to suggest that the association had failed to engage with researchers beyond education or that it was silent when changes in how educational research was funded were proposed at national and European level, this particular campaign around Horizon 2020 saw a qualitative change occur in the type and range of engagement. At the heart of the engagement was the decision by EERA Council to work in partnership with other interested research organisations in the general social science space and beyond under the umbrella of the European Alliance of Social Science and Humanities (EASSH). This ad hoc body sought to marshal the substantial community of social science and humanities researchers in Europe to campaign against the explicit downgrading of this branch of research in the Horizon 2020 programme. Initially focusing on the gathering of signatures through an epetition, the campaign sought to influence, at a political as well as policy level, decision makers in Europe.

Targeting the structure of Horizon 2020, it proposed the creation of a dedicated SOCIAL SCIENCES AND HUMANITIES (SSH) strand and the allocation of substantial funding in this area. In parallel to this was a series of bilateral communications with the Commissioner, the Commission and national governments. Engagement at the national level was channelled through national associations that sought to bring pressure to bear where appropriate and relevant. Council also worked with networks to shape this agenda and to provide alternative routes for influencing policy makers at a national and transnational level. While ultimately unsuccessful, the process did see EERA move into an advocacy role in a way that marked something of a transition from the past. The long-term impact of this decision may be difficult to judge at this juncture but the continued engagement with both funding bodies and the EASSH appears to be significant. The publication of 'EERA's Agenda for Horizon 2020' in September 2013 (EERA, 2013) not only provides a summary of the position of EERA in relation to Horizon but also articulates in a very concise fashion a set of principles around which to further engage with external agencies when addressing issues such as this in the future.

While much of what has been outlined above appears to be relatively uncontroversial, the decision to engage in a process of lobbying and engagement at a political level was seen as being problematic by a significant number of member associations. This was not necessarily due to a disagreement with the ends being sought but rather was a result of long-standing organisational, and at times cultural, understandings as to what is appropriate activity for a research association. For some members of EERA Council, researchers should engage with research and other researchers and stay away from political entanglements. For others, the requirement to engage at a political level is both a core part of their self-definition as educators as well as a necessity in a changing research environment. Discussions around these points enlivened Council meetings for the period leading up to and during the campaign, and while the decisions made clearly came down on one side of the argument vis-à-vis the other, the underpinning tensions remained, and could 
perhaps be seen emerging in a pointed disagreement around political engagement that occurred at the Istanbul Conference and which will be addressed below.

\section{Challenging Conversations}

One of the most impressive aspects of any analysis of EERA is the longevity and flexibility of the association. There have of course been challenges, but it is worth noting that from the perspective of those working on Council at least, these challenges have been addressed in a collegial and positive manner. While acknowledging this, there are a number of issues that could prove problematic in future years that both Council and the broader EERA community might wish to address. Some of these are obvious, and have been referenced in this short review. Issues such as the size and make-up of Council, the relationship between Council, networks and associations, the ever-changing nature of educational research and the external pressures seeking to mould research to particular agendas are obvious. There are other, less obvious challenges, and it is to one of these that we will turn by way of conclusion.

In the course of the 2013 European Conference on Educational Research in Istanbul delegates were faced with a situation of political instability and street protest. Some delegates were caught up in the protests as a result of the location of their hotels, while most attended the conference and did not see much of the protests. There was, however, quite a fractious debate at the Council meeting held in the immediate aftermath of the conference which in essence sought to explore the extent to which it was appropriate for an organisation such as EERA to move beyond educational research to a direct engagement with the political and social realities facing members and member associations. For many at this meeting this issue was seen as being a defining one and was seen as raising the fundamental question as to the function and purpose of EERA. A number of delegates felt it was impossible to engage with educational research in a vacuum, arguing that it was fundamentally a political process, whether this was acknowledged or not. This comes to the forefront especially when EERA brings ECER, with thousands of participants and thereby also economic contributions, to a place where, for example, freedom of expression is suppressed. In this context the idea of acting - to quote one of the delegates - as a quasi- 'Olympic committee claiming that we just do education and that education has nothing to do with politics' was considered to be an untenable position. Drawing from their own history of social and political liberalism in the area of free speech, Council members argued strongly for the moral imperative underpinning educational research and suggesting SUGGESTED that any attempt to remove EERA from this space would be a betrayal of a core function of education in any society.

Arguing equally as forcefully from the opposing position, members of a number of associations warned of the danger of EERA involving itself in internal political and cultural struggles which, almost by definition, were complex, contested and controversial. This position, far from suggesting that there was no moral imperative underpinning educational research, indicated that there were a number of important issues that needed to be addressed and suggested that in addition to the already identified area of free speech, the necessity to respect cultural and political diversity were key elements of any functioning pan-European organisation. One obvious undercurrent in the discussion as it progressed was the danger of straying into the territory of cultural imperialism.

Given the limitations placed on the discussion by the Council format, the conversation ended with a compromise decision to allow the president to frame a response which would go some way to addressing the issue without making a definitive judgment. While useful procedurally, it did not really address the core issues raised, and to an extent they remain unexplored in any real sense. We would argue that in coming months and years EERA may have to examine in a structured, honest and open manner the core values underpinning the organisation. While the issue of political engagement in a time of social conflict was the one that initiated the discussion in Istanbul it is arguable that other situations, and here the decision to engage in political lobbying around Horizon 2020 comes to the fore again, will force the association to explore who it is and what it stands for. A review of the Constitution of EERA provides many interesting insights. While there is a statement relating to charitable status, an acknowledgement of the German tax code and an outline of the rights and responsibilities of members, there is no mention of the values underpinning the 
association. This is not to be critical of EERA. The Constitution as designed met the needs for an association which was seeking to streamline its procedures in a way that would allow it to face the challenges posed by a rapidly expanding membership and a changeable financial context. The real issue now, however, is to decide whether the association needs to move beyond issues related to procedures and governance to ones of values, principles and engagement in a formal sense.

\section{Conclusion}

Among the challenges facing EERA in coming years, the issue of engagement may be one of the more compelling, or it may fade as a priority as other problems become more pressing. To an extent, all a review such as this can do is offer a snapshot of where EERA is now from the perspective of national associations and suggest areas where attention might be needed in future years. In either event, the necessity to consider what EERA is for as it faces its second 20 years will be an ever-present reality but one, we are sure, EERA will address with sensitivity, consideration and a few good dinners!

\section{References}

European Educational Research Association (EERA) (2010) Archives, Networks and Policy. IS THIS A REPORT? CITY AND PUBLISHER OR URL?

European Educational Research Association (EERA) (2011) EERA Constitution. http:/ / www.eeraecer.de/fileadmin/user_upload/Documents/Constitution/Englisch_Constitution_25 062011.pdf

European Educational Research Association (EERA) (2013) EERA’s Agenda for Horizon 2020. http://www.eeraecer.de/fileadmin/user_upload/Documents/EERA_Agenda_H2020_250813.pdf

European Educational Research Association (EERA) (2014) TITLE OF DOCUMENT? http: / www.eeraecer.de/about/council/

JOE O'HARA is Professor of Education and Head of the School of Education at Dublin City University, Ireland. He is Director of the Centre for Educational Evaluation at DCU and his research interests focus on school evaluation and inspection, issues of quality in education and policy development in Initial Teacher Education. He is a member of the Teaching Council of Ireland and was President of the Educational Studies Association of Ireland (ESAI) from 2010 to 2012.Correspondence: joe.ohara@dcu.ie

GUNILLA HOLM, PhD, is a professor of education in the Institute of Behavioural Sciences at the University of Helsinki and director of the Nordic Centre of Excellence in Education 'Justice through Education'. Her research interests are focused on photography as a research method as well as issues in education related to race, ethnicity, class and gender. She has published widely on photography and cultural diversity issues in education as well as on schooling in popular culture. She has co-edited several books, including Contemporary Youth Research: local expressions and global connections (2005) and Schooling in the Light of Popular Culture (1994). Correspondence: gunilla.holm@helsinki.fi 